\title{
PROMOCIÓN DE LOS DERECHOS EN SALUD EN PERÚ: UNA APROXIMACIÓN DESDE LA PERSPECTIVA DE ACCIÓN DE LA SUPERINTENDENCIA NACIONAL DE SALUD
}

\author{
Edward Mezones-Holguín ${ }^{1,2, a}$, Ricardo Díaz-Romero ${ }^{3, b}$, Jackeline Castillo-Jayme ${ }^{3, c}$, María Jerí-de-Pinho ${ }^{3, d}$, \\ Vicente Benites-Zapata ${ }^{1,4, e}$, Edith Marquez-Bobadilla ${ }^{1, f}$, César López-Dávalos ${ }^{5,9}$, Flor de María Philipps-Cuba ${ }^{6,7,9}$
}

\begin{abstract}
RESUMEN
En Perú, la Superintendencia Nacional de Salud (SUSALUD) tiene la responsabilidad de promover, proteger y defender los derechos en salud (DES) de cada peruano. No obstante, la existencia de una cultura deficiente en materia de DES, el escaso conocimiento de la población sobre sus DES, las reducidas acciones de cooperación entre usuarios, prestadores y financiadores, así como, la restringida participación ciudadana, son condiciones frecuentes en el sistema de salud peruano (SSP). Ese contexto ha demandado la puesta en marcha de distintas acciones por parte de SUSALUD, dentro de las cuales se encuentran el desarrollo de las juntas de usuarios de los servicios de salud (JUS) y del Programa de Acciones Integradas para la Promoción de los Derechos en Salud (PAIPDES-SUSALUD). Ambas intervenciones pretenden incrementar el grado de confianza entre la ciudadanía y el Estado, lo cual amplía y fortalece las competencias para el ejercicio de los DES por parte de los ciudadanos. El presente artículo parte con una exposición breve acerca del estado de los conocimientos, actitudes y prácticas de los usuarios de los servicios de salud del SSP respecto a los DES. Luego, se explican de modo global ambos programas, presentándose sus objetivos y pautas generales, una descripción genérica de sus contenidos; asimismo, se exhiben algunos indicadores de proceso y resultado, para, finalmente, ofrecer una discusión y perspectivas sobre el particular. Se considera que la implementación gradual de las JUS y el PAIDPDES-SUSALUD impulsan la consolidación de la participación social en salud en el SSP y contribuyen favorablemente al empoderamiento del ciudadano como constituyente clave en la arena de los DES en Perú.
\end{abstract}

Palabras clave: Derecho a la salud; Participación ciudadana, Organización comunitaria; Calidad de la atención de salud; Reforma del sector salud; Organizaciones del usuario; Perú (Fuente: DECS BIREME).

\section{PROMOTION OF HEALTH RIGHTS IN PERU: AN APPROACH FROM THE PERSPECTIVE OF THE PERUVIAN NATIONAL HEALTH AUTHORITY}

\begin{abstract}
In Peru, The National Health Authority (SUSALUD) is granted the responsibility to promote, protect and defend the health rights of every citizen. However, in the Peruvian Health System (PHS) there exists an insufficient culture of health rights, a low level of knowledge of health rights by the population, and a limited degree of cooperation between users, providers and funders. In light of this limited popular participation in the health rights of the citizenry, it has been necessary for SUSALUD to pursue various activities in order to promote the exercise of health rights by Peruvians. Among the strategies implemented are the development of Organized Boards of Users $(\mathrm{OBU})$ and a Program of Integrated Actions for the Promotion of Health Rights (PIAPHI). The aim of both interventions is to increase the level of trust between citizens and government, which fosters and strengthens the capacity of citizens to pursue their health rights. In this article we begin with a brief presentation on the state of knowledge, attitudes and practices of users of the health system regarding their rights. Then we explain both programs, their goals and procedures, and a general description of their activities. Also, some indicators of process and some results are presented along with discussion and future prospects. We believe that the gradual implementation of the $\mathrm{OBU}$ and PIAPHI programs will enhance the participation of Peruvians in their health system, and will contribute positively to their empowerment and the pursuit of their health rights.
\end{abstract}

Key words: Right to health; Citizen participation; Communitarian organization; Quality of health care; Health care reform; Organized groups of users; Peru (Source: MeSH NLM).

\footnotetext{
Intendencia de Investigación y Desarrollo, Superintendencia Nacional de Salud. Lima, Perú.

Escuela de Medicina, Universidad Peruana de Ciencias Aplicadas. Lima, Perú.

Intendencia de Promoción de Derechos (IPROM), Superintendencia Nacional de Salud. Lima, Perú

Centro de Investigación de Salud Pública, Instituto de Investigación, Facultad de Medicina Humana, Universidad de San Martín de Porres. Lima, Perú. Superintendencia Adjunta de Promoción y Protección de Derechos, Superintendencia Nacional de Salud. Lima, Perú.

Alta Dirección, Superintendencia Nacional de Salud. Lima, Perú.

Escuela de Postgrado, Universidad Peruana de Ciencias Aplicadas. Lima, Perú.

a Médico, maestro en Ciencias en Epidemiología Clínica; ${ }^{\mathrm{b}}$ médico cirujano; ${ }^{\mathrm{c}}$ licenciada en Periodismo; ${ }^{\mathrm{d}}$ licenciada en Trabajo Social; ${ }^{\mathrm{e}}$ médico, maestro en ciencias en Investigación Epidemiológica; ${ }^{\mathrm{f}}$ licenciada en Estadística; ${ }^{\mathrm{g}}$ médico, especialista en Administración de Salud

Recibido: 14/05/2016 Aprobado: 15/06/2016
}

Citar como: Mezones-Holguín E, Díaz-Romero R, Castillo-Jayme J, Jerí-de-Pinho M, Benites-Zapata V, Marquez-Bobadilla E, et al. Promoción de los derechos en salud en Perú: una aproximación desde la perspectiva de acción de la Superintendencia Nacional de Salud. Rev Peru Med Exp Salud Publica. 2016;33(3)520-28. doi:10.17843/rpmesp.2016.333.2302 


\section{INTRODUCCIÓN}

Los derechos en salud (DES) son ineludibles al ser humano (1) y constituyen una condición que debe ser asumida como primordial por la sociedad (2). No obstante, los DES resultan ser uno de los derechos sociales y políticos con mayor dificultad de ejercicio, convirtiéndose en un importante reto para los gobiernos, quienes convienen en significativos esfuerzos dirigidos a garantizarlos ${ }^{(3,4)}$. El sistema de salud peruano (SSP) es fragmentado y segmentado con una importante diversificación prestacional y financiera ${ }^{(5,6)}$. En ese marco, emerge la necesidad de iniciativas que busquen lograr el posicionamiento de los DES en el contexto peruano.

En Perú se han desarrollado diversas aproximaciones para favorecer el ejercicio de los DES. Uno de los hitos fue la promulgación de la Ley Marco de Aseguramiento Universal en Salud (AUS) en el año 2009, con la cual se buscó extender la cobertura de asegurados, ampliar la cartera de beneficios y garantizar la calidad de atención en salud ${ }^{(7)}$. Ese mismo año se promulgó la Ley que establece los derechos de las personas usuarias de los servicios de salud, en la cual los DES se encontraban incluidos, aunque no se explicitaba una institución que los aborde de manera transversal en todo el SSP ${ }^{(8)}$. En el año 2013 se proclaman los lineamientos y medidas de la reforma del sector Salud, donde se reconoce que: La salud es un derecho fundamental y se crea la Superintendencia Nacional de Salud (SUSALUD) como una institución pública autónoma con el propósito de promover, proteger y defender los DES ${ }^{(9)}$, y se le otorga facultades para actuar activamente en el ámbito de los derechos de los usuarios de los servicios de salud en el SSP ${ }^{(10)}$. En consecuencia, SUSALUD se convierte en un organismo público clave llamado a cumplir un rol protagónico en el campo de los DES.

En una sociedad que aspira al bienestar, el el empoderamiento de las personas con los DES es cardinal; sin embargo, ello suele ser deficiente ${ }^{(11,12)}$. Un ciudadano informado es un elemento con alto valor de contribución positiva para un sistema de salud, no solo debido a que sus conocimientos pueden servir de base para ejercer sus derechos, sino también porque puede constituirse en una fuente de participación activa en la mejora del desenvolvimiento del sector ${ }^{(13,14)}$. Empero, existen diversos factores que pueden conllevar a que se adolezca de falta de dicha condición, entre ellos el escaso conocimiento de la población sobre sus DES, una cultura deficiente en materia de DES y la ausencia de instituciones que fomenten la adquisición de estas competencias ${ }^{(15,16)}$. Lo expuesto puede constituir una debilidad social significativa, no obstante, resulta ser, al mismo tiempo, una oportunidad de mejora abordable mediante intervenciones efectivas $y$ eficientes ${ }^{(17,18)}$. La situación aquí descrita no es ajena a la realidad de países en vías en desarrollo como Perú.
En ese marco, SUSALUD desarrolla diferentes acciones en virtud de promover los DES con especial énfasis en la generación de un ambiente favorable para lograr una óptima participación ciudadana. Acorde al mandato de su creación, SUSALUD cuenta con una estructura orgánica y un dinamismo funcional dirigidos a lograr un abordaje integral de los DES ${ }^{(9)}$. Una de las dependencias que la conforman es la Intendencia de Promoción de Derechos en Salud (IPROM), la cual es el órgano de línea responsable de establecer, implementar, conducir y promover las actividades orientadas a fortalecer el ejercicio de los DES. Ello implica un trabajo plural, transparente, desconcentrado y multidisciplinario que requiere la articulación con otros órganos dentro de la Superintendencia, así como la alianza con otras organizaciones. Todo ello busca el posicionamiento del ciudadano como elemento clave en la arena de los DES.

En el presente artículo se expone de modo sucinto el desarrollo de las juntas de usuarios de los servicios de salud (JUS) y del Programa de Acciones Integradas para la Promoción de los Derechos en Salud (PAIPDESSUSALUD). Inicialmente, se muestra información sobre los usuarios de los servicios de salud y los DES en Perú. Luego, se realiza una descripción global de lo desarrollado, se puntualizan los objetivos y pautas generales de las intervenciones, se muestran algunos indicadores de proceso y resultado y, por último, se despliega una discusión y perspectivas sobre cada programa.

\section{DERECHOS Y SERVICIOS DE SALUD: EVIDENCIA PRIMARIA EN PERÚ}

En Perú, se han evaluado los conocimiento, percepciones y prácticas de los usuarios de los servicios de salud con respecto a sus derechos al acceso a los servicios de salud, al acceso a la información, a la atención y recuperación de la salud, al consentimiento informado, así como, a la protección de los derechos en salud; aspectos reconocidos en la normatividad peruana vigente $^{(8,10)}$. En la Encuesta Nacional de Satisfacción de Usuarios en Salud del año 2015 (ENSUSALUD) -desarrollada por el Instituto Nacional de Estadística e Informática y SUSALUD con una muestra representativa nacional- se evidenció una baja proporción de usuarios de la consulta médica ambulatoria con conocimiento adecuado y con percepción de cumplimiento pleno de sus derechos (Figura 1). Adicionalmente, como proxy a la protección de los DES, en esa misma encuesta se observó que $11,4 \%$ de los usuarios había tenido problemas en la atención en los últimos seis meses; sin embargo, solo $22,6 \%$ presentó un reclamo y, de estos, $16,6 \%$ refirió haber recibido la solución total del mismo. Estos hallazgos plantean la presencia de notorias insuficiencias en el ejercicio de los DES por parte de los ciudadanos peruanos. 


\section{EL PROGRAMA JUNTAS DE USUARIOS EN SALUD}

Existe una situación de restringida participación ciudadana en el campo de los DES asociada a múltiples causas. Una de ellas es la ausencia de un canal institucionalizado que articule las iniciativas de los usuarios con las acciones estatales en el sector. Asimismo, existen reducidas acciones de colaboración entre los usuarios, instituciones prestadoras de servicios de salud (IPRESS) e instituciones administradoras de fondos de aseguramiento en salud (IAFAS) para mejorar los servicios de salud. En ese contexto, SUSALUD tiene el mandato de promover la participación y vigilancia ciudadana, por lo que asume el liderazgo en la conformación de las JUS de acuerdo a lo estipulado en la legislación peruana.

\section{OBJETIVOS PLANTEADOS Y PAUTAS GENERALES}

Las JUS son un espacio de articulación trilateral entre SUSALUD, los usuarios, y las IAFAS e IPRESS, que busca incrementar el conocimiento sobre DES, efectivizar el uso de mecanismos para su ejercicio y mejorar los servicios de salud. Las JUS incorporan las opiniones del ciudadano, y fomentan su participación activa más allá de los niveles clásicos de información y consulta. De esta manera, se complementa el enfoque de la gestión pública desde el Estado hacia la sociedad y viceversa, a través de la retroalimentación mutua bajo una orientación territorial, de género, intercultural e intergeneracional. En ese contexto, la institucionalización de la participación -lo que implica contar con normas y presupuesto específicos- es crítico para la sostenibilidad y crecimiento del programa. No obstante, ello no supone una sustitución del rol de SUSALUD por parte de las JUS.

\section{DISEÑO Y DESCRIPCIÓN GENERAL}

La conformación de la JUS cuenta con la IPROM en calidad de Secretaría Técnica, y contempla la inclusión de ciudadanos, agrupados y no agrupados, que participan en los procesos de elección y selección, respectivamente. En el primer caso, los representantes provienen de las asociaciones de pacientes, conformadas por aquellas personas que se reúnen en relación a una enfermedad o discapacidad en común, y las agrupaciones por etapas de ciclo de vida, constituidas por grupos de jóvenes y grupo de adultos mayores. Dentro de los no agrupados se encuentran los usuarios de los servicios de salud de la Seguro Social en Salud (EsSalud), Seguro Integral de Salud, comunidades locales de administración en salud, sanidades de las Fuerzas Armadas y de la Policía Nacional del Perú, seguros privados, usuarios con discapacidad no agrupados y usuarios no asegurados que se atienden en los servicios de Salud.

Este trabajo acoplado permite ampliar los canales con el objeto de recoger las apreciaciones e iniciativas de los usuarios, informar acerca de los mecanismos para ejercer los DES y para encaminar los casos de posible vulneración de los DES a la Intendencia de Protección de Derechos de SUSALUD (IPROT). Asimismo, las JUS permiten desarrollar acciones de evaluación de los servicios de salud a través de la información obtenida por parte de los usuarios. Todo ello se plasma en el Plan de Trabajo Anual, el instrumento central en la gestión concertada de la JUS. El programa tiene cuatro fases:

La fase de concepción y diseño se realizó en el segundo semestre del año 2013 con la creación de la Secretaría Técnica, para luego, delinear su estructura y funcionamiento con participación de las organizaciones

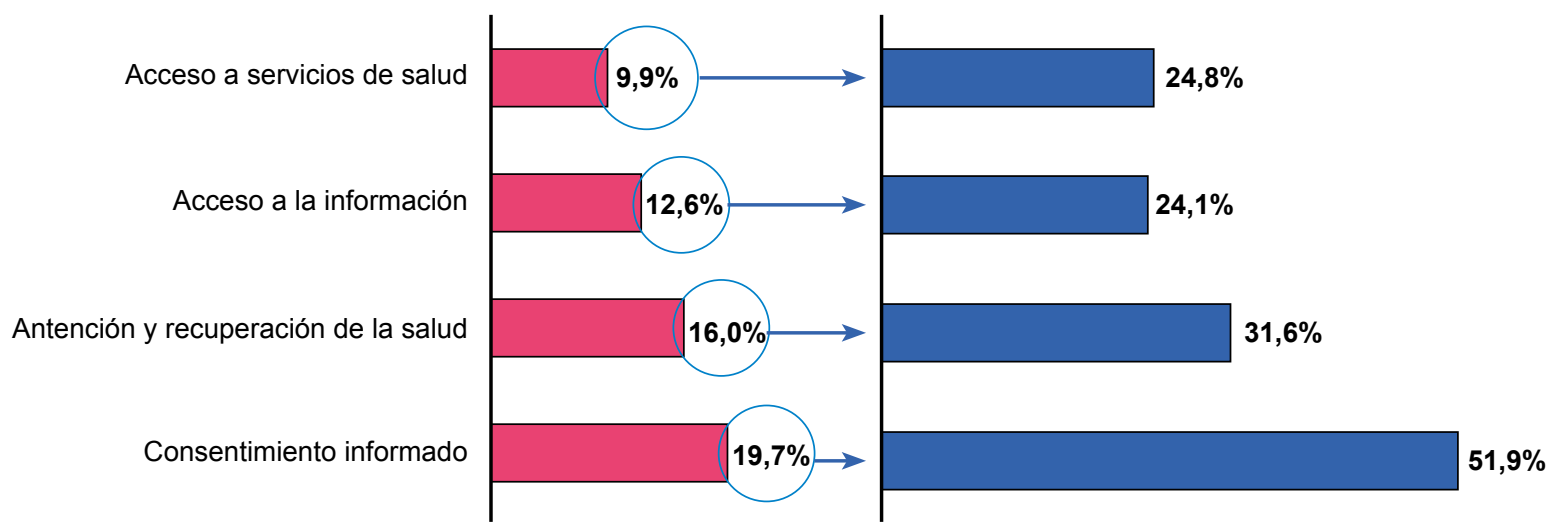

Conocimiento adecuado sobre DES

Percepción sobre cumplimiento pleno de los DES

Figura 1. Nivel de conocimiento adecuado y percepción sobre el cumplimiento pleno de los derechos en salud (DES) por parte de los usuarios de la consulta externa a nivel nacional (ENSUSALUD 2015) 
de usuarios de servicios de salud y con instituciones reguladoras nacionales de servicios públicos, lo cual incluyó la revisión de otras experiencias de participación ciudadana tanto nacionales como internacionales. Este diseño fue validado mediante metodología cualitativa con ciudadanos de la Costa, Sierra y Selva de Perú, específicamente de las regiones de Loreto, Lambayeque, Ayacucho y Lima. Posteriormente, se elaboraron el reglamento de elecciones para las organizaciones de usuarios, la guía para la selección aleatoria de usuarios no agrupados y el reglamento para el funcionamiento de las JUS. SUSALUD asume los costos para la conformación, funcionamiento y ejecución de las acciones de la JUS, mientras que la participación de los miembros es ad honorem.

La fase de implementación central se inició en marzo del año 2014, concentrándose en la creación de la JUS en la capital peruana. Se convocaron a dos procesos electorales en esta región, uno dirigido a organizaciones de usuarios de servicios de salud, y otro a los usuarios no organizados, quienes fueron seleccionados de manera aleatoria con un aplicativo diseñado y validado por la Intendencia de Investigación y Desarrollo de SUSALUD (IID). Ello estuvo acompañado de actividades de difusión acerca de los DES, SUSALUD y los mecanismos de participación ciudadana, y de una convocatoria en medios de comunicación. Los ciudadanos seleccionados fueron contactados por distintas vías. Para los comicios se contó con la colaboración de la Oficina Nacional de Procesos Electorales (ONPE), con la participación de la Asociación Civil Transparencia en calidad de veedores, así como, un notario público y un fedatario informático juramentado. Tras los procesos, los miembros electos fueron juramentados y presentados públicamente ante las distintas organizaciones de la región. Posterior a ello, se mantuvieron sesiones de planeamiento y evaluación participativa para elaborar el plan anual de trabajo de la JUS de manera concertada con SUSALUD.

La fase de implementación regional corresponde a la réplica a otras regiones de lo desarrollado en la implementación central. Desde su inicio el año 2015, este proceso se ha realizado en seis regiones: Callao, Cusco, Loreto, Lambayeque, Ayacucho y Arequipa. Ante el hecho de no poseer oficinas físicas de SUSALUD en cuatro de ellas (la región Callao tiene como base las oficias de SUSALUD en Lima, y durante el año 2015 se creó la Intendencia Macrorregional Norte con sede en Lambayeque), se optó por la conformación de equipos itinerantes de la IPROM y la construcción de las Redes Regionales de Apoyo a las Juntas de Usuarios de Servicios (REDAJUS), integradas por gobiernos regionales y locales, universidades, colegios profesionales, entre otras instituciones. Además, se utiliza material audiovisual con testimonios de lo realizado en Lima como instrumentos de reproducción.
Actualmente, se cuenta con espacio virtual para las JUS en la web de SUSALUD (http://portales.susalud.gob.pe /web/portal/jus).

En la fase de monitoreo se desarrollan sesiones ordinarias de la JUS (cuatro al año como mínimo), para evaluar avances de la ejecución del plan, fortalecer su organización interna, e intercambiar información sobre acciones de protección de los DES; y reuniones bilaterales entre la Junta y la Secretaría Técnica, para retroalimentar la relación de cooperación entre ambos. Asimismo, posteriormente se efectuarán mediciones de impacto.

\section{RESULTADOS}

La Tabla 1 presenta algunos indicadores del proceso de implementación de la intervención, alcanzados en términos de frecuencia absoluta. Lo descrito refleja el carácter desconcentrado de la propuesta, se incluye consecuciones relacionadas a entrenamiento y fortalecimiento de las competencias de los miembros en calidad de atención, en DES, mecanismos para ejercerlos, y habilidades para difundirlos. También se expresan algunos alcances sobre la promoción del trabajo cooperativo y la elaboración de propuestas formales de mejora (específicamente en el Hospital Sergio Bernales, al norte de la ciudad de Lima).

\section{EL PROGRAMA DE ACCIONES INTEGRADAS EN LA PROMOCIÓN DE LOS DERECHOS EN SALUD}

La construcción de una cultura para el ejercicio de los DES es trascendental para el Estado. Ello pone de manifiesto la asunción de su rol de garante de derechos y su accionar para el fortalecimiento de un sistema de salud de calidad al servicio de sus ciudadanos. Sin embargo, la ausencia y fragilidad de esta cultura es frecuente en el SSP, lo cual conlleva al crecimiento de casos de vulneración de derechos. Su explicación estriba, entre otros factores, en la precaria situación de competencias sobre DES por parte de los usuarios, ello como manifestación de las escasas capacidades del conjunto de actores que integran el sistema en la promoción del ejercicio de los DES. A ello se suman los limitados espacios adecuados para la difusión de los DES que posibiliten el contacto directo entre ciudadanos, usuarios, IPRESS, IAFAS y otros protagonistas. Otro elemento es el bajo seguimiento $y$ monitoreo a las acciones de prestadores y financiadores de servicios sobre el funcionamiento de sus plataformas de atención al usuario de los servicios de salud (PAUS). En respuesta a lo mostrado, y dado el potencial origen multifactorial de la situación, SUSALUD desarrolla el PAIPDES-SUSALUD. 
Tabla 1. Resumen de los principales logros de las juntas de usuarios de servicios de salud (JUS) en su trabajo cooperativo con la Superintendencia Nacional de Salud (SUSALUD) y otros actores del sistema de salud

\begin{tabular}{|c|c|c|c|}
\hline Rubro & Indicador & $\begin{array}{l}\text { Frecuencia } \\
\text { (Al 2015) }\end{array}$ & Observaciones \\
\hline $\begin{array}{l}\text { Institucionalización de la } \\
\text { participación de los usuarios en } \\
\text { SUSALUD }\end{array}$ & Número de JUS & 7 & $\begin{array}{l}\text { Una en la región Lima y seis réplicas } \\
\text { en las regiones: Callao, Cusco, Loreto, } \\
\text { Lambayeque, Ayacucho y Arequipa }\end{array}$ \\
\hline \multirow{2}{*}{$\begin{array}{l}\text { Capacitación a los miembros de } \\
\text { la junta de usuarios }\end{array}$} & Número de talleres realizados & 22 & $\begin{array}{l}\text { Con las JUS de Lima, Callao, Arequipa, } \\
\text { Ayacucho, Cusco, Lambayeque y Loreto }\end{array}$ \\
\hline & $\begin{array}{l}\text { Número de miembros de la JUS } \\
\text { capacitados }\end{array}$ & 200 & $\begin{array}{l}\text { Con las JUS de Lima, Callao, Arequipa, } \\
\text { Ayacucho, Cusco, Lambayeque y Loreto }\end{array}$ \\
\hline \multirow{2}{*}{$\begin{array}{l}\text { Campañas conjuntas entre la } \\
\text { JUS y SUSALUD sobre los DES }\end{array}$} & $\begin{array}{l}\text { Número de campañas } \\
\text { realizadas }\end{array}$ & 14 & $\begin{array}{l}\text { Con las JUS de Lima, Callao, Loreto y } \\
\text { Ayacucho }\end{array}$ \\
\hline & $\begin{array}{l}\text { Número de orientaciones } \\
\text { realizadas }\end{array}$ & 8644 & $\begin{array}{l}\text { Con las JUS de Lima, Callao, Loreto y } \\
\text { Ayacucho }\end{array}$ \\
\hline $\begin{array}{l}\text { Elaboración de propuestas para } \\
\text { mejorar los servicios de salud. }\end{array}$ & $\begin{array}{l}\text { Número de propuestas } \\
\text { elaboradas }\end{array}$ & 1 & $\begin{array}{l}\text { Con la JUS de Lima en el Hospital Sergio } \\
\text { Bernales }\end{array}$ \\
\hline $\begin{array}{l}\text { Reuniones con instituciones } \\
\text { públicas para la difusión de la } \\
\text { junta de usuarios }\end{array}$ & $\begin{array}{l}\text { Número de reuniones entre la } \\
\text { JUS e instituciones públicas }\end{array}$ & 8 & $\begin{array}{l}\text { Con IPRESS e IAFAS de Lima } \\
\text { Metropolitana. Donde además se dieron } \\
\text { las bases para el desarrollo de un trabajo } \\
\text { conjunto * }\end{array}$ \\
\hline
\end{tabular}

DES: derechos en salud.

IPRESS: instituciones prestadoras de servicios de salud.

IAFAS: instituciones administradoras de fondos de aseguramiento en salud

* En el caso de las IAFAS se incluyeron al Seguro Social del Perú (EsSalud), al Seguro Integral de Salud y al Fondo de Aseguramiento en Salud de la Policía Nacional del Perú (SALUDPOL). En el caso de las IPRESS fueron el Instituto Nacional de Enfermedades Neoplásicas, el Instituto Nacional de Salud del Niño de San Borja, el Hospital Edgardo Rebagliati Martins, el hospital Sergio Bernales, así como, las comunidades locales de administración de salud de Villa Libertad, Villa Alejandro, Nuevo Lurín y Julio C. Tello.

\section{OBJETIVOS PLANTEADOS Y PAUTAS GENERALES}

EI PAIPDES-SUSALUD pretende afianzar y fortificar las competencias para la promoción del ejercicio de los DES en los ciudadanos, usuarios y operadores del SSP. Para ello, una de las bases principales es la interacción, participación y cooperación entre los actores que contribuyan a la construcción de una cultura de protección de los DES. De modo específico, estas acciones se dirigen a la construcción de habilidades cognitivas y actitudinales para la coordinación y cooperación interinstitucional entre entidades públicas, privadas y de la ciudadanía a nivel local, y en ese accionar proporciona para la promoción del ejercicio de los DES y sobre el rol de SUSALUD para su protección. A ello se adiciona el reforzar el aprendizaje para el cumplimiento de los verificadores de funcionamiento de las PAUS por parte de las IPRESS e IAFAS mediante el despliegue de acciones de acompañamiento. Además, se procura establecer espacios para la información y orientación sobre el ejercicio de los DES y los respectivos mecanismos para ello, con lo cual se propicia la interacción entre los actores del SSP. Finalmente, se realiza la difusión de estas actividades a través de medios de comunicación masivos en cada una de las regiones del país.

\section{GENERACIÓN Y DESCRIPCIÓN GENERAL DEL PROGRAMA}

EI PAIPDES-SUSALUD es resultado de un proceso gradual que inicia el año 2013 con actividades de difusión a través de campañas informativas a la ciudadanía en general, así como reuniones de trabajo con IPRESS e IAFAS -públicas y privadas- en Lima y Callao; y que se extiende a inicios del 2014 con la adición de la vigilancia a las PAUS. Es así que, con la integración de estas tres acciones, sumado a una campaña de difusión en medios regionales de comunicación masiva, se generó una intervención cuyo destino inicial fueron seis regiones del Perú: Lima, Junín, Loreto, Moquegua y San Martín.

Posteriormente, en el último tramo del año 2014, el programa es perfilado para extender su accionar hacia las 25 regiones del Perú. El público objetivo fue la ciudadanía, los usuarios de los servicios de salud y las IPRESS e IAFAS tanto públicas como privadas. Asimismo, se cuenta con la participación del Ministerio de Trabajo y Promoción del Empleo, las direcciones regionales de salud (DIRESA), las gerencias regionales de salud (GERESAS), las JUS, los gobiernos locales tanto a nivel provincial como distrital, y el trabajo cooperativo con los medios masivos de comunicación. La 
implementación inicial se efectuó entre febrero y diciembre del 2015. Para el año 2016 se han diferenciado y priorizado públicos, a la vez que está en proceso de validación de metodologías para medir el impacto en grupos específicos.

EI PAIPDES-SUSALUD cuenta con un equipo itinerante multidisciplinario conformado por comunicadores, médicos y abogados de IPROM que acuden a cada una de las regiones del Perú. Por lo general, se requiere de aproximadamente 25 días para lograr la implementación de una jornada regional del PAIPDES-SUSALUD: 15 días destinados a las coordinaciones previas, cinco a la ejecución en sí y cinco días posteriores a la evaluación y certificación de las IAFAS e IPRESS participantes. Específicamente, en la realización de cada visita regional son cuatro los momentos identificados:

El momento de construcción, consiste en reuniones de coordinación con entidades estatales, instituciones privadas y de la sociedad civil, donde se expone el rol de SUSALUD, los objetivos de la visita y la importancia del trabajo cooperativo. A este nivel se identifican los actores locales, y se establecen acuerdos para la ejecución de las actividades.

El momento de sensibilización, incluye sesiones de trabajo con IAFAS e IPRESS donde se socializan, mediante metodología activa, aspectos relacionados al marco legal, a las funciones de SUSALUD, a las PAUS y vigilancia ciudadana; asimismo, con dichas entidades se realiza un acompañamiento previo y posterior a la jornada central como una estrategia de monitoreo.

El momento de consolidación, corresponde al desarrollo de ferias informativas sobre DES y participación ciudadana, el cual se dirige a los ciudadanos y cuenta con la participación de IAFAS e IPRESS. A este nivel se concreta un espacio físico de interacción entre todos los actores del SSP. Adicionalmente, se comparte información sobre los DES, sus mecanismos de ejercicio y la trascendencia de la participación de los ciudadanos en la mejora de la atención de los servicios de salud con énfasis en la problemática local de vulneración de DES identificada.

Momento de difusión, se refiere a la utilización de los medios de comunicación masiva para dar a conocer el programa y sus mensajes, se realizan entrevistas, notas periodísticas y ruedas de prensa en diversas fases de la intervención.

En la Figura 2 se presenta una esquematización de los momentos que conforman la intervención, más como objeto pedagógico, pues existe un interactuar dinámico entre ellos.

\section{RESULTADOS}

Para valorar la intervención se disgregaron las mediciones en seis grandes rubros, acorde con los objetivos trazados y las actividades realizadas. Los primeros cinco muestran indicadores de proceso: información y orientación a los ciudadanos, capacitación a prestadores y financiadores, acciones de acompañamiento a prestadores y financiadores, coordinación y cooperación entre actores, difusión en medios de comunicación masiva. El último corresponde a un indicador de resultado mediante la frecuencia de consultas, quejas y reclamos por parte de los ciudadanos.

La Tabla 2 comparte un resumen y se realiza un análisis comparativo de lo ocurrido en una etapa previa a la ejecución de PAIPDES-SUSALUD (2013-2014) frente al consolidado anual tras su implementación (2015), se observa una mejora substancial de los indicadores.

\section{DISCUSIÓN Y PERSPECTIVAS}

La participación ciudadana es un elemento capital en un sistema de salud. Se reconoce que existen diferencias marcadas en la concepción de la participación en salud y la interacción con los servicios de salud entre los diversos actores del sistema ${ }^{(19)}$. Bajo dichas circunstancias, las JUS se sitúan como un programa imparcial, transparente e inclusivo que ha logrado cambiar terrenos que usualmente era de confrontación por ambientes de cooperación que permite la obtención de mejoras en los servicios de salud y en el grado de confianza entre la ciudadanía y el Estado. En Perú, las JUS como tal, son un modelo singular que no tiene precedente en el sector Salud. Sin embargo, se encuentra aún en una etapa de implementación progresiva a nivel nacional, por lo que este artículo recoge la experiencia hasta este nivel.

Si bien en América Latina existen algunas iniciativas de participación ciudadana en salud, estas difieren del modelo peruano de las JUS. Las juntas de salud en Costa Rica se centralizan en el desarrollo de un trabajo administrativo bajo un modelo de gestión compartida de cada establecimiento de salud (20). En Colombia existen los comités de participación comuntaria que incluyen la participación de agentes sociales y el Estado, y las asociaciones de usuarios que reúnen a los afiliados al sistema de seguridad social para asesorar a sus miembros, velar por la calidad de los servicios y la defensa del usuario ${ }^{(21,22)}$. El aval ciudadano es una estrategia vigente en México, por la cual una persona, grupo o institución académica se incorpora a vigilar el proceso de mejora de los servicios de salud, con el objeto de participar como "garante de un buen servicio de salud" (23). Todas las experiencias descritas contrastan con las JUS, bien por cumplir un rol administrativo o fiscalizador, por ser de alcance local, por encontrarse circunscrito a un financiador o prestador específico, o por carecer de un espacio institucionalizado por el símil de SUSALUD en los respectivos países, entre otras características. 
Tabla 2. Resumen de los principales resultados encontrados en el Programa de Acciones Integradas de Promoción de los Derechos en Salud (PAIPDES-SUSALUD)

\begin{tabular}{|c|c|c|c|c|}
\hline Rubro & Indicador & $\begin{array}{l}\text { Preintervención } \\
(2013-2014)^{*}\end{array}$ & $\begin{array}{l}\text { Posintervención } \\
(2015) \dagger\end{array}$ & Incremento \\
\hline \multirow{2}{*}{$\begin{array}{l}\text { Información y orientación a } \\
\text { los ciudadanos }\end{array}$} & $\begin{array}{l}\text { Número de ferias informativas } \\
\text { realizadas }\end{array}$ & 16 & 32 & $100 \%$ \\
\hline & $\begin{array}{l}\text { Número de orientaciones a ciudadanos } \\
\text { en DES }\end{array}$ & 7775 & 20463 & $163,2 \%$ \\
\hline \multirow{3}{*}{$\begin{array}{l}\text { Capacitación a prestadores y } \\
\text { financiadores }\end{array}$} & Número de capacitaciones realizadas & 12 & 29 & $141,7 \%$ \\
\hline & $\begin{array}{l}\text { Número de IPRESS } \S \text { e IAFAS que } \\
\text { participan en capacitaciones }\end{array}$ & 294 & 831 & $182,7 \%$ \\
\hline & $\begin{array}{l}\text { Número de directivos y encargados de } \\
\text { PAUSS informados en DES y sobre } \\
\text { funcionamiento de PAUSS }\end{array}$ & 576 & 1422 & $146,9 \%$ \\
\hline \multirow[t]{2}{*}{$\begin{array}{l}\text { Acciones de acompañamiento } \\
\text { a prestadores y financiadores }\end{array}$} & $\begin{array}{l}\text { Número de acciones de } \\
\text { acompañamiento para funcionamiento } \\
\text { de PAUSS }\end{array}$ & 533 & 578 & $8,5 \%$ \\
\hline & $\begin{array}{l}\text { Número de encargados de atención } \\
\text { al usuario que orientan en campañas } \\
\text { informativas sobre derechos y deberes } \\
\text { en salud }\end{array}$ & $\begin{array}{c}479 \text { (De } 108 \\
\text { IPRESS e IAFAS) }\end{array}$ & $\begin{array}{l}766 \text { (De } 294 \\
\text { IPRESS IAFAS) }\end{array}$ & $59,9 \%$ \\
\hline \multirow[t]{2}{*}{$\begin{array}{l}\text { Coordinación y cooperación } \\
\text { entre actores }\end{array}$} & $\begin{array}{l}\text { Número de colaboradores de } \\
\text { instituciones públicas en orientación a } \\
\text { ciudadanos }\end{array}$ & $\begin{array}{c}138 \text { (De } 26 \\
\text { instituciones) }\end{array}$ & $\begin{array}{l}272 \text { (De } 56 \\
\text { instituciones) }\end{array}$ & $97,1 \%$ \\
\hline & $\begin{array}{l}\text { Número de organizaciones de } \\
\text { usuarios orientan a ciudadanos en } \\
\text { Ferias informativas sobre derechos y } \\
\text { deberes en salud }\end{array}$ & 0 & 3 & $3 \ddagger$ \\
\hline $\begin{array}{l}\text { Difusión en medios masivos } \\
\text { de comunicación }\end{array}$ & $\begin{array}{l}\text { Número de medios masivos de } \\
\text { comunicación dan a conocer mensajes } \\
\text { del programa }\end{array}$ & 20 & 184 & $820,0 \%$ \\
\hline $\begin{array}{l}\text { Consultas, quejas y reclamos } \\
\text { por parte de ciudadanos }\end{array}$ & $\begin{array}{l}\text { Consultas, interposiciones de } \\
\text { buenos oficios y quejas por parte de } \\
\text { ciudadanos }\end{array}$ & $21786 \S$ & $64714 \S$ & $197,0 \%$ \\
\hline \multicolumn{5}{|c|}{$\begin{array}{l}\text { * En ocho (8) regiones } \\
\text { † En veinticinco (25) regiones } \\
\text { † Valor absoluto } \\
\text { § A nivel nacional } \\
\text { DES: derechos en salud } \\
\text { IPRESS: instituciones prestadoras de servicios de salud } \\
\text { IAFAS: instituciones administradoras de fondos de aseguramiento en salud } \\
\text { PAUSS: plataforma de atención al usuario de los servicios de salud }\end{array}$} \\
\hline
\end{tabular}

Para el año 2017 se estima la continuación de las réplicas de la JUS de Lima en las otras regiones, previa valoración de criterios técnicos, económicos y sociales. Simultáneamente durante esta etapa se llevarán a cabo acciones preparatorias para la conformación de un espacio de participación de los usuarios en el nivel nacional que complementa la participación en el nivel regional y local. Asimismo, se debe puntualizar que en junio de 2016 la JUS de Lima cumplió dos años de funcionamiento, por lo que se lleva a cabo un proceso electoral dirigido a reemplazar a la mitad de sus miembros.

Por su parte, el PAIPDES-SUSALUD constituye un proceso de recreación sucesivo que rescata los aprendizajes desarrollados y que los actualiza de acuerdo a las demandas de los potenciales usuarios.
Con el programa se ha producido la extensión de la perspectiva técnica y gerencial, con la incorporación del enfoque de derechos y el ejercicio de ciudadanía, donde los servicios de salud se conviertan en espacios de dignificación de las personas ${ }^{(24)}$. Todo ello considera también que el disfrute pleno de la salud, depende del compromiso y voluntad de cada individuo y del cuerpo social, por lo que la accesibilidad, disponibilidad, aceptabilidad y calidad de los servicios de salud será efectivo en tanto se aseguren procesos de desarrollo de capacidades del Estado como garante de derechos y del ciudadano como titular de los mismos ${ }^{(25-27)}$.

Si se mantiene esta línea de acción, resulta imperioso aplicar medidas innovadoras producto de las lecciones recogidas durante el periodo transcurrido desde el 
inicio de su implementación. Para una nueva etapa del PAIPDES-SUSALUD se plantea un programa de énfasis educativo-comunicacional que amplíe la movilización de otros actores, entre ellos, los propios colaboradores de SUSALUD, las organizaciones sociales de base, y las instituciones educativas de nivel básico y superior. Asimismo, se incorporan nuevas metodologías que desde las dimensiones lúdica y artística; establezcan un proceso progresivo que culmina en el intercambio de experiencias de los distintos actores en la promoción de los DES.

Se evidencia que la implementación de las dos intervenciones muestra indicadores positivos tanto a nivel de proceso como resultado. Por ejemplo, los hallazgos reflejan un aumento de casi tres veces del número de quejas y reclamos por parte de los usuarios de los servicios de salud, lo cual podría ser el reflejo de una mejora de los niveles de empoderamiento de los ciudadanos cuando sienten que se vulneran sus DES. No obstante, a este punto del tiempo, la ausencia de estimaciones de impacto es una limitación del presente análisis, por lo que su cálculo se convierte en una tarea pendiente de alta trascendencia. A ello debe sumarse que el documento no se desarrolla alrededor del concepto del derecho fundamental a la salud estipulado en la Constitución de Organización Mundial de la Salud, donde se le define como el "goce del máximo grado de salud que se puede lograr" (28), sino que se encauza en los derechos de los usuarios de los servicios de salud y al accionar propio de SUSALUD, de acuerdo al cuerpo normativo peruano ${ }^{(8-10)}$.

\section{CONCLUSIONES}

En el contexto de la promoción y protección de los DES, tanto las JUS como el PAIPDES-SUSALUD son programas que propugnan el accionar mancomunado de múltiples sectores estatales, estamentos privados y de la sociedad civil. La implementación de estas intervenciones favorece el desarrollo de alianzas entre diversos sectores (Salud, Educación, Trabajo, Justicia, entre otros), niveles de gobierno (nacional, regional y local), financiadores, prestadores y usuarios; todo ello con el fin ulterior de obtener como beneficio mayor el acceso del usuario a una atención de calidad. Las dos iniciativas se encuentran en franco proceso de crecimiento y mejora continua; en la actualidad, los indicadores de proceso y resultado son positivos, no obstante, es pertinente y necesario realizar mediciones de impacto a mediano plazo.

Contribuciones de autoría: EMH, RDR, JCJ, MJP, FPC, CLD realizaron contribuciones substanciales en la concepción del artículo. EMH, VBZ, FPC, realizaron contribuciones substanciales en el diseño del artículo. RDR, JCJ, MJP, CLD

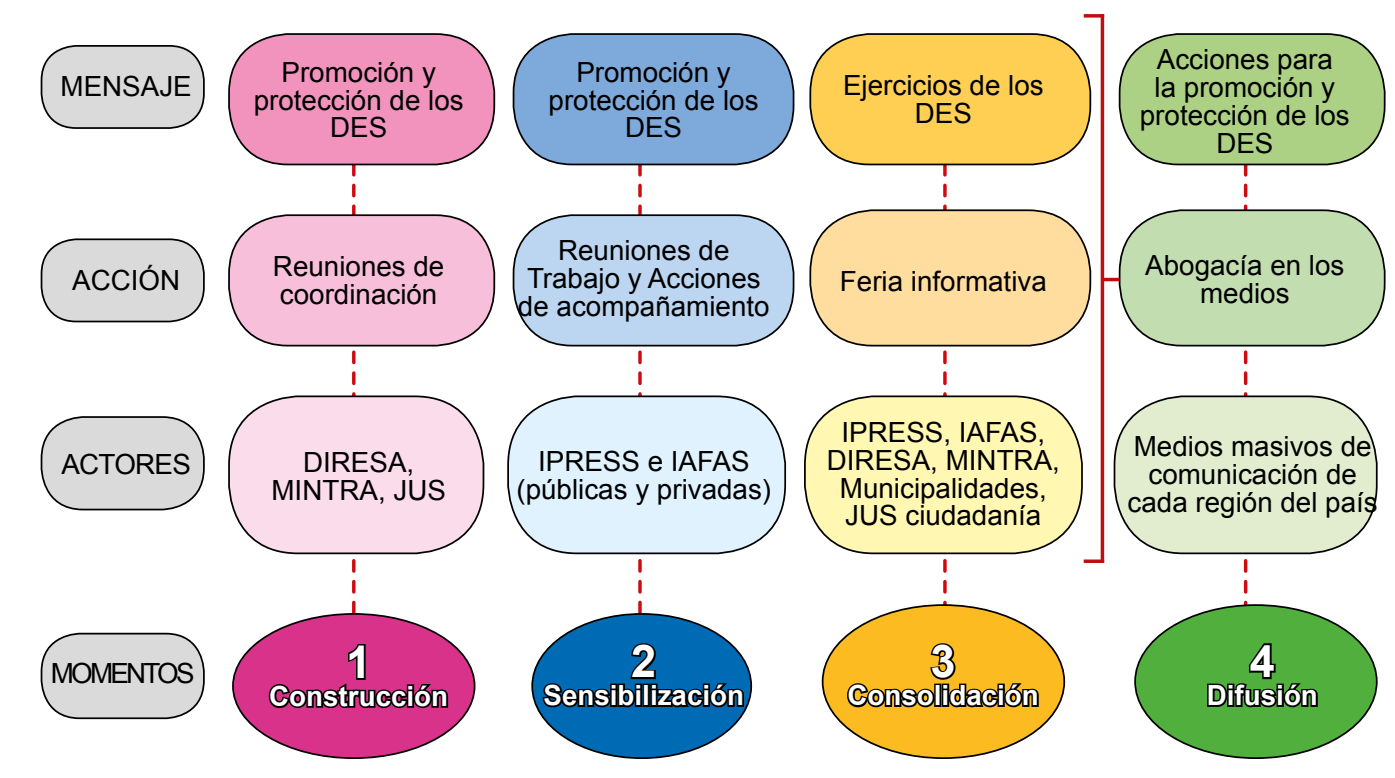

DIRESA: direcciones regionales de salud

MINTRA: Ministerio de Trabajo y Promoción del Empleo

JUS: juntas de usuarios en salud

IPRESS: instituciones prestadores de servicios de salud

IAFAS: instituciones administradoras de fondos de aseguramiento en salud.

Figura 2. Estructura de la intervención de las jornadas regionales del Programa de Acciones Integradas para la Promoción de los Derechos en Salud (PAIPDES-SUSALUD) 
realizaron la adquisición de datos. EMH, VBZ, EMB realizaron el análisis de datos, EMH, RDR, JCJ, MJP, VBZ, EMB, CLD, FPC realizaron la interpretación de datos. EMH, JCJ, MJP, FPC redactaron el primer borrador del artículo. EMH, RDR, JCJ, MJP, BVZ, EMB, CLD, FPC realizaron una revisión crítica que proporciona un importante contenido intelectual. Todos los autores aprobaron la versión final del artículo y están de acuerdo en ser responsables de todos los aspectos, lo que implica asegurar de que todas las preguntas relacionadas a la validez e integridad de cualquier parte del artículo han sido apropiadamente investigadas y resueltas.

Fuente de financiamiento: el presente artículo fue financiado por la Superintendencia Nacional de Salud (SUSALUD)

Conflictos de interés: al momento de la publicación los autores laboran en la Superintendencia Nacional de Salud (SUSALUD).

\section{REFERENCIAS BIBLIOGRÁFICAS}

1. Gruskin S, Mills EJ, Tarantola D. History, principles, and practice of health and human rights. Lancet. 2007;370(9585):449-55.

2. Eleftheriadis P. A right to health care. J Law Med Ethics. 2012;40(2):268-85. doi: 10.1111/j.1748-720X.2012.00663.x

3. Norheim OF. Ethical priority setting for universal health coverage: challenges in deciding upon fair distribution of health services. BMC Med. 2016;14(1):75. doi: 10.1186/s12916-016-0624-4.

4. Atun R, de Andrade LO, Almeida G, Cotlear D, Dmytraczenko T, Frenz P, et al. Health-system reform and universal health coverage in Latin America. Lancet. 2015;385(9974):1230-47. doi: 10.1016/ S0140-6736(14)61646-9.

5. Alcalde-Rabanal JE, Lazo-González O, Nigenda G. Sistema de salud de Perú. Salud Publica Mex. 2011;53 Suppl 2):s243-s54.

6. Eibenschutz C, Saco A, Tamez S, Zafra $\mathrm{X}$, Ramírez R. Considerations on health reform process (1993-2013) and social participation in Peru. Saude Debate. 2014;38(103):872-82.

7. Wilson L, Velásquez A, Ponce C. La Ley Marco de Aseguramiento Universal en Salud en el Perú: análisis de beneficios y sistematización del proceso desde su concepción hasta su promulgación. Rev Peru Med Exp Salud Publica. 2009;26(2):207-17.

8. Perú, Congreso de la República. Ley 29414. Ley que Establece los Derechos de las Personas Usuarias de los Servicios de Salud. (2 de octubre de 2009).

9. Perú, Presidencia de la República. Decreto Legislativo $\mathrm{N}^{\circ} 1158$. Decreto Legislativo que dispone de medidas destinadas al fortalecimiento y cambio de denominación de la Superintendencia Nacional de Aseguramiento en Salud. (6 de diciembre de 2013).

10. Perú, Ministerio de Salud. Decreto Supremo 027-2015-SA. Aprueban Reglamento de la Ley que establece los Derechos de las Personas Usuarias de los Servicios de Salud. (13 de agosto de 2015)
11. Frankish CJ, Kwan B, Ratner PA, Higgins JW, Larsen C. Challenges of citizen participation in regional health authorities. Soc Sci Med. 2002;54(10):1471-80.

12. Zúñiga-Fajuri A. Teorías de la justicia distributiva: una fundamentación moral del derecho a la protección de la salud. Convergencia. 2011;18(55):191-211.

13. Church J, Saunders D, Wanke M, Pong R, Spooner C, Dorgan M. Citizen participation in health decision-making: past experience and future prospects. J Public Health Policy. 2002;23(1):12-32.

14. Thurston WE, MacKean G, Vollman A, Casebeer A, Weber M, Maloff B, et al. Public participation in regional health policy: a theoretical framework. Health Policy. 2005;73(3):237-52.

15. Cotlear D, Gómez-Dantés O, Knaul F, Atun R, Barreto IC, Cetrángolo O, et al. Overcoming social segregation in health care in Latin America. Lancet 2015;385(9974):1248-59. doi: 10.1016/ S0140-6736(14)61647-0.

16. Honarvar B, Lankarani KB, Rostami S, Honarvar F, Akbarzadeh A, Odoomi N, et al. Knowledge and Practice of People toward their Rights in Urban Family Physician Program: A PopulationBased Study in Shiraz, Southern Iran. Int J Prev Med. 2015;6:46. doi: 10.4103/2008-7802.158172.

17. Abelson J, Forest P-G, Casebeer A, Mackean G, Effective Public Consultation Project Team. Will it make a difference if I show up and share? A citizens' perspective on improving public involvement processes for health system decision-making. J Health Serv Res Policy. 2004;9(4):205-12.

18. Singer MA. Community participation in health care decision making: is it feasible? CMAJ. 1995;153(4):421-4.

19. Delgado Gallego ME, Vázquez Navarrete ML, Zapata Bermúdez Y, Hernán García M. Participación social en salud: conceptos de usuarios, líderes comunitarios, gestores y formuladores de políticas en Colombia. Una mirada cualitativa. Rev Esp Salud Publica. 2005;79(6):697-707.
20. Ruiz AI. La Democracia Local y la participación de las juntas de salud. Revista Costarricense de Trabajo Social. 2003;14(1):12-23.

21. Echeverry M. Las organizaciones de usuarios: incidiendo en la construcción del derecho a la salud. Rev Gerenc Polit Salud. 2009;8(17):80-105.

22. Vázquez ML, Siqueirab E, Kruzeb I, Da Silva A, Leiteb IC. Los procesos de reforma y la participación social en salud en América Latina. Gac Sanit. 2002;16(1):30-8.

23. Nigenda-López GH, Juárez-Ramírez C, Ruiz-Larios JA, Herrera CM. Participación social y calidad en los servicios de salud: la experiencia del aval ciudadano en México. Rev Saude Pública. 2013;47(1):44-51.

24. Franco-Giraldo A, Alvarez-Dardet C. Derechos humanos, una oportunidad para las políticas públicas en salud. Gac Sanit. 2008;22(3):280-6.

25. Arrivillaga M, Borrero YE. Visión comprensiva y crítica de los modelos conceptuales sobre acceso a servicios de salud, 1970-2013. Cad Saude Pública. 2016;32(5):e00111415. doi: 10.1590/0102-311X00111415.

26. Braveman PA, Kumanyika S, Fielding J, Laveist T, Borrell LN, Manderscheid R, et al. Health disparities and health equity: the issue is justice. Am J Public Health. 2011;101 Suppl 1:S149-55. doi: 10.2105/ AJPH.2010.300062.

27. Ruger JP. Rethinking equal access: agency, quality, and norms. Glob Public Health. 2007;2(1):78-96. doi: $10.1080 / 17441690600911480$.

28. Organización Mundial delaSalud(OMS). Constitución de la Organización Mundial de la Salud. Ginebra: OMS; 1948.

Correspondencia: Edward Mezones-Holguin Dirección: Avenida Velasco Astete 1398, Santiago de Surco, Lima, Perú.

Teléfono: (+511)1372614 anexo: 5412

Correo electrónico:emezones@susalud.gob.pe/ emezones@gmail.com 